\title{
Effect of non surgical periodontal therapy on glycemic control in patients with type II diabetes mellitus: Case control study
}

\author{
Lt Col Vijayalaxmi V Malali ${ }^{1}$, Maj Satisha T S2, \\ Lt Col A K J ha ${ }^{3}$, Col S K Rath
}

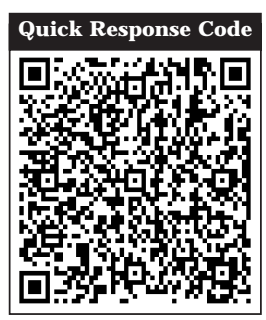

doi: $10.5866 / 2015.7 .10003$

1,2,3\&44 Command Military Dental Centre

(Central Command)

Lucknow-226002

\section{Article Info:}

Received: J anuary 10, 2015

Review Completed: February 9, 2015

Accepted: March 10, 2015

Available Online: April, 2015 (www.nacd.in)

(C) NAD, 2015 - All rights reserved

\section{Email for correspondence:}

viju_chandu@yahoo.com

\begin{abstract}
:
AI M: The purpose of the present study was to assess the effect of non-surgical periodontal therapy on glycemic control of type II diabetes patients with generalized chronic periodontitis.

MATERI ALS AND METHODS: A total of 40 patients were enrolled in this case control study, the selected patients were divided in to two groups. Group I (cases)- consisting of patients diagnosed with type II diabetes with generalized chronic periodontitis and Group II (controls) - consisting of non-diabetics with generalized chronic periodontitis. The patients who qualified the study were rescheduled for laboratory, periodontal and clinical examinations. Periodontal parameters and glycosylated haemogl obin (A1C) were evaluated at baseline and 3 months.

RESULTS: All periodontal parameters improved significantly in Group I. Thereis a highly significant differencein the pretreatment and 03 months post NSPT Hblac values in group I ( $<<0.0001)$, a non significant differencein group II ( $p>0.05), A 1 C$ levels decreased statistically significantly more in the cases versus the controls independently of other confounders.

CONCLUSION: This study provides evidence that periodontal treatment contributes to improved glycemic control in type II diabetes mellitus patients. Larger controlled trials are needed to confirm if this finding is generalizable to other populations of patients with type II diabetes.
\end{abstract}

Key words: diabetes mellitus; glycaemic control; HbAlc; nonsurgical; periodontal disease.

\section{INTRODUCTION:}

Diabetes mellitus is a clinically and genetically heterogeneous group of metabolic disorders manifested by abnormally high levels of glucose in the blood. The impact of diabetes mellitus on the oral cavity has been well researched. ${ }^{1}$ Examination of the available data reveals strong evi-dence that diabetes is a risk factor for gingivitis and periodontitis, and the level of glycemic control appears to be an important determinant in this relation-ship. Epidemiologic studies in diabetic adults have often shown an increase in extent and severity of periodon-titis. The relationship between metabolic control of dia-betes and periodontal

\section{INDIAN JOURNAL OF Dental Advancements}

Journal homepage: www. nacd. in 
disease is difficult to define con-clusively. Research suggests that this association is similar to the association between glycemic control and the classic complications of diabetes such as ret-inopathy and nephropathy. Thus, although poor control of diabetes clearly increases the risk of diabetic complications, there are many poorly controlled diabetic individuals without major complications. ${ }^{2}$ Conversely, good control of diabetes greatly decreases the risk of diabetic complications. Periodontal diseases are now recognized as the sixth complication of diabetes mellitus. ${ }^{3}$

Although the bulk of oral health-related research pertaining to diabetes has focused on the impact of diabetes on periodontal health, there is also evidence to support the notion that periodontal infections contribute to problems related to glycemic control. A two-way relationship between periodontal disease and diabetes mellitus has been proposed. ${ }^{4}$ The biochemical basis where by hyperglycemia may lead to the microvascular complications seen in diabetes is the increased accumulation of advanced glycation end products (AGEs) in the plasma and tissues of diabetic patients. Binding AGE- modified proteins to macrophage receptors initiates a cycle of cytokine upregulation, with synthesis mostly of IL-1 and TNF $\alpha$. Periodontal inflammation mediated-cytokine synthesis and secretion may amplify the magnitude of the AGE mediated cytokine response and vice-versa.

In a large epidemiologic study in the U nited States, adults with poorly controlled diabetes had a 2.9-fold increased risk of having periodontitis compared to non-diabetic adult subjects; conversely, well-controlled diabetic subjects had no significant increase in the risk of periodontitis. ${ }^{5}$ In a crosssectional study of pa-tients who had type I diabetes for a mean duration of over 16 years, subjects with poor glycemic control had more interproximal attachment loss and bone loss than well-controlled subjects. ${ }^{3}$ Similar results have been found in other studies in which the percent-age of deep periodontal pockets and the preval ence of severeattachment loss increased as the glycaemic control worsened. ${ }^{6,7}$ The objective of the present study was to perform a clinical and metabolic comparison of the response to nonsurgical periodontal therapy between type II diabetic patients and non-diabetic patients.

\section{MATERIAL AND METHODS:}

A total of 40 patients were enrolled in this clinical study after taking informed consent. The selected patients were divided into two groups, Group I (cases)- consisting of patients diagnosed with type II diabetes with generalized moderate chronic periodontitis and Group II (controls) consisting of non-diabetics with generalized moderate chronic periodontitis.

The following werecriteria for inclusion of these individuals in the study, age group of 35-60 years, presence of type II diabetes, clinical diagnosis of generalized moderate chronic periodontitis defined by loss of clinical attachment of $4-6 \mathrm{~mm}$ in all quadrants and presence of minimum 10 teeth per arch and no modification in medication in the last 02 months before or during the study. Exclusion criteria were as follows: presence of systemic disease that could influence the course of the periodontal disease, intake of antibiotics or anti-inflammatory drugs in the past 04 weeks before study, current smokers or ex- smokers for < 05 years and pregnancy.

The patients who qualified the study were rescheduled for laboratory, periodontal, and clinical examinations. They underwent an initial examination including a general medical history, and periodontal examination consisting following parameters at baseline and 03 months following scaling and root planing : bleeding on probing, oral hygieneindex-simplified (OHI-S), probing depth and clinical attachment level. Probing depth and clinical attachment level were recorded using a Williams periodontal probe at six sites per tooth (mesial, central, and distal; buccally as well as lingually/ palatally) using a customized acrylic stent as a reference to determine the site and angle of measurements, ensuring reproducibility during examinations. Blood samples weretaken at baseline and at 03 months recall visit to monitor glycated hemogl obin.

A specific treatment protocol was designed for the study. All patients received standard oral instructions before the first session of scaling and root planing. All patients received scaling and root 
planing in 2 sittings spaced within 24 -36 hours using standard periodontal currettes and ultrasonic device.

\section{STATISTICAL ANALYSIS:}

The mean and standard deviation values of the parameters were calculated and grouped per subject per group. The changes in bleeding on probing between the baseline and the 3 month post non surgical periodontal therapy (NSPT) were tested within the treatment groups using Wilcoxon test. The Mann-Whitney test was used to determine significant differences between the groups.

\section{RESULTS:}

There is a highly significant difference in the pre treatment and 03 months post NSPT Hblac values in group I $(p<0.0001)$, a non significant difference in group II ( $p>0.05)$ (Table 1,Figure 1). There is no significant difference in the pre treatment and 03 months post NSPT BOP between group I and group II ( $>>0.05)$, and a highly significant difference between the pre treatment and 03 months post NSPT BOP in group I and group II $(p<0.0001)$. There is no significant difference in the pre treatment and 03 months post NSPT OHIS scores between group I and group II $(p>0.05)$, and a highly significant difference between the pre treatment and 03 months post NSPT OHIS scores in group I and group II $(p \measuredangle 0.0001)$. There is a highly significant difference in the pre treatment and 03 months post NSPT probing depth values in group I and group II $(p<0.0001)$, and no significant difference between the pre treatment and 03 months post NSPT probing depth values in group I and group II ( $p>0.05$ ) (Table 2, Figure 2). There was no significant difference between the pretreatment and 03 months post NSPT CAL in group I and group II) (Table 3, Figure 3].

\section{DISCUSSION:}

In the present study, two groups (diabetic and non diabetic) with generalized moderate chronic periodontitis were selected and treated by non surgical periodontal therapy to determine whether there were clinical and laboratory differences between them following periodontal treatment. The results of the present study show that non surgical periodontal treatment of type II diabetic patients with generalized moderate periodontal disease is associated with significant improvement in their glycemic control after 03 months. All the periodontal clinical parameters (BOP, OHI-S, PPD, CAL) were significantly improved in the group I. Results in the literature regarding the influence of periodontal therapy on glycemic control in diabetes are generally contradictory. ${ }^{8,9}$ The effectiveness of periodontal therapy on glycemic control and systemic inflammation is not proven beyond doubt and studies should be interpreted with caution because they may differ in design, population type (type I or II diabetic patients or both)severity, and extent of periodontal disease (healthy, gingivitis and different degrees of periodontitis), length of study period (adequate or not to register variations in $\mathrm{HbAlc}$ levels), or treatment type (scaling and root planning alone or associated with use of local and systemic antibiotics in addition to scaling and root planning) among other variables. ${ }^{10}$

In the present study, antibiotics were not used as an adjunctive to non-surgical periodontal disease treatment. Administration of antibiotics, especially systemic, can lead to controversial results regarding $\mathrm{HbA1C}$, because they may affect other systemic sources of infection/inflammation and potential reduction in $\mathrm{HbA} 1 \mathrm{C}$ levels cannot be sol ely attributed to the local reduction in the infectious/inflammatory periodontal burden. Furthermore, it is well accepted that $\mathrm{HbAlC}$ is affected by systemic inflammation. ${ }^{11}$, 12 Most studies examining the effect of periodontal disease treatment in patients with diabetes have incorporated antibiotic administration (topical or systemic). 8,9 The results of the present study, concerning the glycemic effect of non-surgical periodontal therapy (without any local or systemic antibiotic administration) in diabetes, are in accordance with those of Kiran et al, who on 44 Turkish patients with type 2 diabetes and moderate periodontal disease, showed such treatment provided a statistically significant reduction in periodontal parameters and $\mathrm{HbA} 1 \mathrm{C}$ levels after 3 months. ${ }^{13}$

The biologic rationale and the mechanisms underlying the effect that periodontal treatment has on glycemic control and chronic inflammation are not clarified completely, but there is evidence to support 
Table 1: Comparison of Glycated Hemoglobin in diabetic and non-diabetic group

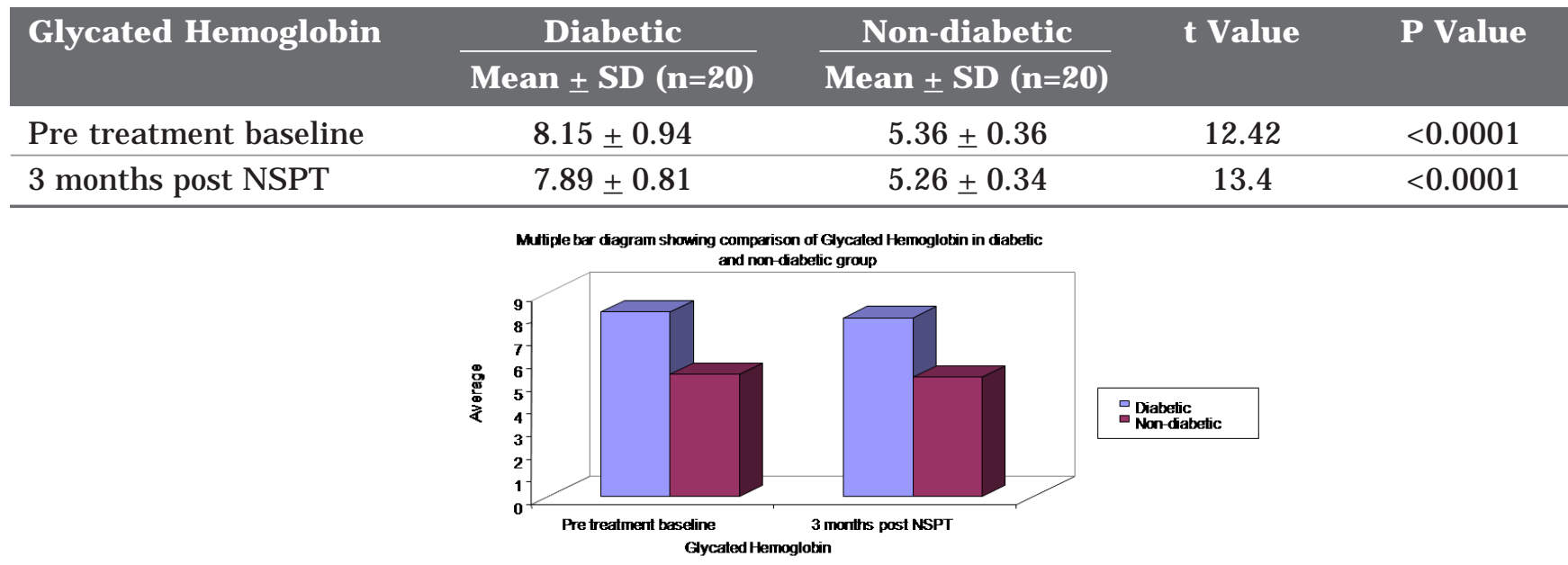

Table 2: Comparison of probing depth in diabetic and non-diabetic group

\begin{tabular}{|c|c|c|c|c|}
\hline Probing depth (mm) & $\frac{\text { Diabetic }}{\text { Mean } \pm \text { SD }(n=20)}$ & $\frac{\text { Non-diabetic }}{\text { Mean } \pm \text { SD }(n=20)}$ & t Value & P Value \\
\hline Pre treatment baseline & $6.85 \pm 1.23$ & $6.6 \pm 1.47$ & 0.59 & $>0.05$ \\
\hline 3 months post NSPT & $5.4 \pm 1.39$ & $5.35 \pm 1.57$ & 0.11 & $>0.05$ \\
\hline
\end{tabular}

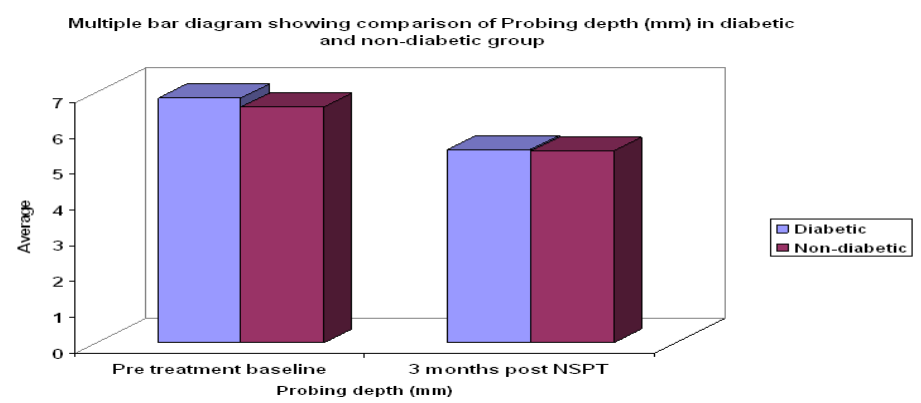

Table 3: Comparison of clinical attachment level in diabetic and non-diabetic group

\begin{tabular}{|c|c|c|c|c|}
\hline CAL (mm) & $\frac{\text { Diabetic }}{\text { Mean } \pm \text { SD }(n=20)}$ & $\frac{\text { Non-diabetic }}{\text { Mean } \pm \text { SD }(n=20)}$ & t Value & P Value \\
\hline Pre treatment baseline & $6.85 \pm 1.23$ & $6.6 \pm 1.47$ & 0.59 & $>0.05$ \\
\hline 3 months post NSPT & $6.85 \pm 1.23$ & $6.6 \pm 1.47$ & 0.59 & $>0.05$ \\
\hline
\end{tabular}

Multiple bar diagram showing comparison of CAL $(\mathrm{mm})$ in diabetic and non-
diabetic group

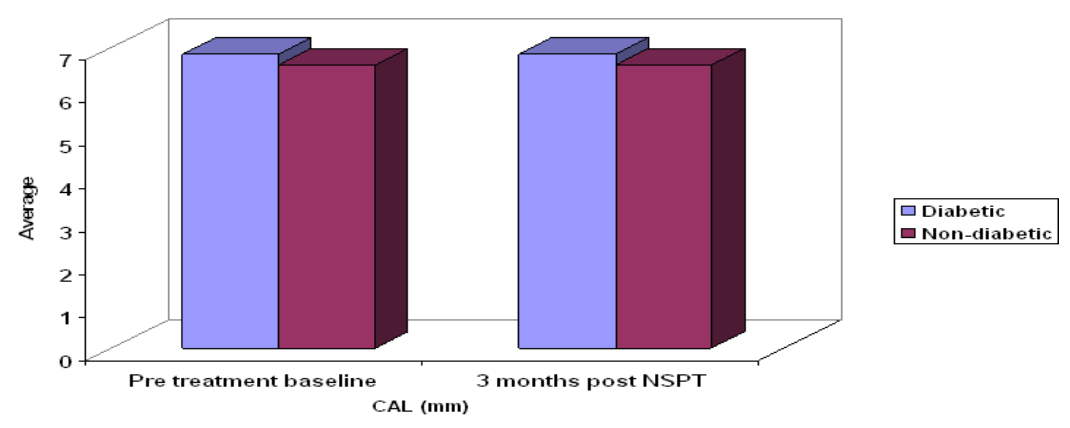


the hypothesis that resolution of inflammation from the periodontal tissues has a favorable effect on $\mathrm{HbA1C}$ levels. The present study was not designed to investigate the underlying mechanisms through which periodontal treatment results in improvement of glycemic control. It is clear that more studies are needed to clarify these mechanisms. The small sample sizeand short follow up period are the major limitations of the present study. Case-control design although provides a higher strength of evidence as compared to other cross-sectional studies, is still Iaden with biases. In evaluating the clinical significance of the data, it must beremembered that although means are excellent summary statistics, they do not directly relate to what is measurable with a periodontal probe. Further prospective randomized control studies with larger sample size, longer follow up period, different populations and additional biochemical parameters are needed to substantiate our findings generalize them to other populations of patients with type II diabetes.

\section{CONCLUSION:}

The present study has demonstrated that non surgical periodontal treatment can improve the periodontal status of patients with type II diabetes and have a favorable effect on glycemic control in these patients.

\section{REFERENCES:}

1. Mealey B L. World Workshop in Clinical Periodontics. Periodontal implication: medically compromised patients. Ann Periodontol 1996;1:256-321
2. Papapanou PN. World Workshop in Clincal Periodontics. Periodontal disease: Epidemiology. Ann Periodontol 1996; 1:1-36.

3. Loe H. Periodontal disease: The sixth complication of diabetes mellitus. Diabetes Care 1993; 16:329-334.

4. Grossi S G, Genco R. Periodontal disease and diabetes mellitus: A two way relationship. Ann Periodontol 1998; 3:51-61.

5. Tsai C, Hayes C, Taylor GW. Glycemic control of type II diabetes and severe periodontal disease in the U.S. adult population. Community Dent Oral E pide-miol 2002; 30:182192.

6. Safkan-Seppala B, Ainamo J. Periodontal conditions in insulin-dependent diabetes mellitus. J Clin Peri-odontol 1992; 19:24-29.

7. Tervonen $\mathrm{T}, \mathrm{K}$ nuuttila $\mathrm{M}$. Relation of diabetes control to periodontal pocketing and alveolar bone level. Oral Surg Oral Med Oral Pathol 1986; 61:346-349.

8. J anket S J, Wightman A, Baird A E, Van DykeT E, J ones J A. Does periodontal treatment improve glycemic control in diabetic patients? Ameta-analysis of intervention studies. J Dent Res 2005; 84:1154-1159.

9. Teeuw W J, Gerdes, V E, Loos B G. Effect of periodontal treatment on glycemic control of diabetic patients: a systematic review and metaanalysis. Diabetes Care 2010; 33:421-427.

10. Salvi G E, Carollo-Bittel B, Lang N P. Effects of diabetes mellitus on periodontal and periimplant conditions: update on associations and risks. J Clin Periodontol 2008; 35:398409.

11. Moutsopoulos N M, Madianos P N. Low grade inflammation in chronic infectious diseases: paradigm of periodontal infections. Ann N Y Acad Sci 2006; 1088:251-264.

12. Shoelson SE, LeeJ, Goldfine A B. Inflammation and insulin resistance. J Clin Invest 2006; 116:1793-1701.

13. Kiran M, Arpak N, Unsal E, Erdogan M F. The effect of improved periodontal health on metabolic control in type 2 diabetes mellitus. J Clin Periodontol 2005; 32:266-272.

\section{Gain quick access to our journal online View our journal at wwW.nacd.in}

